\title{
Magnetized Iron Atmospheres for Neutron Stars
}

\author{
Mohan Rajagopal $^{1}$, Roger W. Romani ${ }^{2}$ \\ Dept. of Physics, Stanford University, Stanford CA 94305-4060 \\ and M. Coleman Miller ${ }^{3}$ \\ Dept. of Ast. and Astrophys., University of Chicago, Chicago, IL 60637
}

\begin{abstract}
Using a Hartree-Fock formalism, we estimate energy levels and photon cross sections for atomic iron in magnetic fields $B \sim 10^{13} \mathrm{G}$. Computing ionization equilibrium and normal mode opacities with these data, we construct LTE neutron star model atmospheres at $5.5<\log \left(T_{\text {eff }}\right)<6.5$ and compute emergent spectra. We examine the dependence of the emergent spectra on $T_{\text {eff }}$ and $B$. We also show the spectral variation with the angle between the magnetic field and the atmosphere normal and describe the significant limb darkening in the X-ray band. These results are compared with recent detailed computations of neutron star $\mathrm{H}$ model atmospheres in high fields and with low field $\mathrm{Fe}$ and $\mathrm{H}$ model atmospheres constructed from detailed opacities. The large spectral differences for different surface compositions may be discernible with present X-ray data; we also note improvements needed to allow comparison of Fe models with high quality spectra.
\end{abstract}

\section{Introduction}

Neutron star surface emission has long been sought as a measure of the star's thermal history and, hence, a probe of the equation of state of matter at supra-nuclear densities. Since temperatures remain near $10^{6} \mathrm{~K}$ for $\sim 10^{6} \mathrm{y}$, this emission is best probed in the soft X-ray band. Recent detections of a number of neutron stars with ROSAT (Becker 1995) show good promise of enabling a study of neutron star cooling. While spectra are still generally fit with blackbodies at present, it has been shown (Romani 1987) that radiation transfer through the surface layers can have very significant effects on the emergent spectrum, depending on the composition.

Conditions on the neutron star surface are very uncertain. While the supernova explosion has a mass cut in the iron layer, envelope fallback, accretion during neutron star evolution, and

\footnotetext{
${ }^{1}$ mohan@astro.stanford.edu

${ }^{2}$ Alfred P. Sloan Fellow; rwr@astro.stanford.edu

${ }^{3}$ Compton GRO Fellow; miller@gamma.uchicago.edu
} 
possibly spallation by energetic magnetospheric particles can introduce light elements (eg. $\mathrm{H}$ and $\mathrm{He})$ to the neutron star surface. Because gravitational sedimentation is rapid, the lightest element present should dominate the photosphere and the emergent spectrum. This uncertainty makes it important to compute emergent spectra for both light element and heavy element atmospheres. As shown by Romani (1987) the general trend is for light elements, with Kramer's law opacities at high E, to have emergent spectra with large excesses in the Wien tail over the equivalent blackbody. Heavy element atmospheres give spectra much closer to blackbodies, but show strong line and edge features. Recent updates of low B model atmosphere computations (Rajagopal \& Romani 1996 (RR96); Zavlin, Pavlov \& Shibanov 1997 (ZPS96)) provide a good set of models for comparison with low field neutron stars. As an example, RR96 found that H spectra fit ROSAT PSPC observations of the low-B millisecond pulsar J0437-4715 much better than did heavy element atmosphere spectra.

Most observed neutron stars, however, have surface fields at least as strong as the surface dipole, $10^{12.5} \mathrm{G}$ or higher. Detailed treatment of the atomic cross sections and the equation of state has allowed Pavlov and colleagues (Pavlov et al. 1995; Shibanov et al. 1992) to study emergent spectra from magnetized $\mathrm{H}$ atmosphere neutron stars. Unlike the recycled pulsars, though, the young, high field neutron stars have presumably experienced little accretion. It is therefore important to estimate the spectral effects of heavy element atmospheres to compare with the magnetic $\mathrm{H}$ results. A first attempt was pursued by Miller (1992) who obtained approximate energy levels and cross sections for heavy atoms in strong fields (Miller \& Neuhauser 1991) and computed emergent spectra. These opacities were very approximate, employing only bound-free cross sections and averaging the polarization modes. Pavlov et al. (1995) argue that these approximations are too severe for the high field models.

In this paper we present substantially improved magnetic Fe atmospheres, incorporating more detailed opacity and equation of state estimates, normal mode radiative transfer and more realistic magnetic geometries. These approximate atmospheres will provide a useful baseline for comparison with the magnetic H results. Since fully detailed Fe atmospheres will be very difficult to construct, we focus on the broad spectral differences between models and note the areas where more detailed models can improve the spectral estimates.

\section{Atoms in a High Magnetic Field}

When the electron cyclotron radius $\hat{\rho}=2.5 \times 10^{-10}\left(B / 10^{12} \mathrm{G}\right)^{-1 / 2} \mathrm{~cm}$ becomes smaller than the approximate zero-field outermost electron orbital sizes of an ion $a \approx a_{0}\left(Z_{\text {eff }}+1\right)^{-1}$, $\left(a_{0}=5.2 \times 10^{-9} \mathrm{~cm}, Z_{\text {eff }}\right.$ the net charge of the ion), the ion's atomic structure perpendicular to the field is altered significantly. For hydrogenic atoms of nuclear charge $Z_{\text {eff }}+1$ in the ground state, this occurs at a magnetic field $B=\left(Z_{\text {eff }}+1\right)^{2} B_{c}$, where $B_{c}=2.35 \times 10^{9} \mathrm{G}$. Well below this field the electron wave functions are approximately spherical, whereas far above it they are best represented as cylinders given by $\psi_{m \nu}(z, \rho, \phi)=f_{n m \nu}(z) \Phi_{n m}(\rho, \phi)$, where $(\rho, \phi)$ are cylindrical 
co-ordinates about the magnetic field direction $\hat{z}, \Phi_{n m}(\rho, \phi)$ are the cylindrical Landau states, and $f_{n m \nu}(z)$ is the wave function in the direction of the field. This assumption of separability in cylindrical co-ordinates is sometimes called the 'adiabatic approximation'. Here $\nu$ and $m$ are the $z$ and $\phi$ quantum numbers, and $n$ is the Landau level. The $\Phi_{0 m}(\rho, \phi)$ wave functions are annular, with radius $a_{\perp}=\sqrt{2 m+1} \hat{\rho}$ and width $\sim \hat{\rho}$ (e.g Meszaros 1992).

To compute energies and electron configurations for iron in strong magnetic fields, we use a multiconfigurational Hartree-Fock code developed by Neuhauser, Langanke, \& Koonin (1986; see also Miller \& Neuhauser 1991). This code computes the wave functions and state energies in the 'adiabatic approximation', with Landau level $n=0$ only, since the first Landau energy (cyclotron energy, $E_{c}=11.6 B_{12} \mathrm{keV}$ for a magnetic field of $10^{12} B_{12} \mathrm{G}$ ) greatly exceeds both $k T$ and all the photon energies $E_{\gamma}$ we consider. For the innermost electron of iron at the magnetic fields we consider, $B=10^{12.5} \mathrm{G}$ and $B=10^{13} \mathrm{G}$, this code gives binding energies that can be low by as much as $15 \%$ because the Coulomb field of the nucleus is too strong for the separability assumption. However, for electrons with binding energies less than $\sim 3 \mathrm{keV}$, whose transitions are most important for the observed spectrum, the code gives individual orbital energies accurate to better than $1 \%$. For each ionization state, we have computed the energies and electron configurations of the ground state, and the first several $(\sim 10)$ excited states which generally extend to $>5 k T$ for the typical local atmosphere conditions. As electrons are added to the bare nucleus, in the ground state they occupy the "tightly-bound" $\nu=0$ states, filling $m=0,1,2, \ldots$ in order. These states have no $\hat{z}$ node, and hence are localized nearer the nucleus than the "loosely-bound" $\nu>0$ states. Ground states with many electrons also fill the $\nu=1, m=0,1$ orbitals, with only one electron per orbital (a spin flip requires $\delta E \sim E_{c}$ ). The excited states we have are all formed by promoting the outermost electrons to higher $m$, which costs little energy because of the weak $m$ dependence of $a_{\perp}$ (above). We use these configurations to calculate the bound-free absorption cross section in each of the $(+,-, z)$ polarization modes (described in $\S 4.1$ ) as a function of frequency for each individual electron, for at least the lowest five energy states of each ion. We also calculate the energies of the most important configurations accessible from the ground state via dipole allowed transitions, and some characteristic oscillator strengths for these, for transition energies up to $10 \mathrm{keV}$. These configurations are made by promoting any one of the outermost (large- $m$ ) electrons to $\nu>0$.

\section{Ionization Equilibrium and the Equation of State}

Let the $r^{\text {th }}$-ionized atom $\left(Z_{\text {eff }}=r\right)$ have excited states $k$ with energy levels $E_{r, k}$. The ionization energies are $\chi_{r} \equiv E_{r, 0}-E_{(r-1), 0}$, while the excitation energies are $\epsilon_{r, k} \equiv E_{r, k}-E_{r, 0}$. Then ionization equilibrium in a strong magnetic field gives the ion densities $n_{r}$ as

$$
n_{r}=2 \frac{n_{(r-1)}}{n_{e}}\left(\frac{m_{e} k T}{2 \pi \hbar^{2}}\right)^{3 / 2} \frac{\eta_{r}}{\sinh \eta_{r}} \frac{\sinh \eta_{(r-1)}}{\eta_{(r-1)}} \frac{\eta_{e}}{\tanh \eta_{e}} e^{-\chi_{r} / k T} \frac{\sum_{k=0}^{\infty} e^{-\epsilon_{r, k} / k T}}{\sum_{k=0}^{\infty} e^{\epsilon_{(r-1), k} / k T}}
$$


(Khersonskii 1987), where the sums are over the excitation states of the individual ions. Here $\eta_{e} \equiv \hbar \omega_{B} / 2 k T$ and $\eta_{r} \equiv \hbar \omega_{r} / 2 k T$, where $\omega_{B}=e B / m_{e} c$ is the electron cyclotron frequency, $\omega_{r}=r e B / m_{F e} c$ is the cyclotron frequency of the $r^{t h}$-ionized atom, $m_{e}$ is the mass of the electron, and $m_{F e}$ is the mass of an iron nucleus. The sums are the partition functions for each ion, and the relative abundance of each excitation state is given simply by its Boltzmann factor divided by that sum. At high densities in our atmospheres, the electron continuum energy can be significantly depressed; we approximate this depression by decreasing the ionization energies by $E_{\text {dep }}=(3 / 5) e^{2}\left(4 \pi \rho / 3 m_{F e}\right)^{1 / 3}\left(\left\langle Z_{\text {eff }}^{2}\right\rangle /\left\langle Z_{\text {eff }}\right\rangle\right)=11.8 \rho^{1 / 3}\left(\left\langle Z_{\text {eff }}^{2}\right\rangle /\left\langle Z_{\text {eff }}\right\rangle\right) \mathrm{eV}$, with $\rho$ the atmosphere density in $\mathrm{g} / \mathrm{cm}^{3}$ (Cox \& Giuli 1968). Since we do not have an exhaustive list of $\epsilon_{r, k}$ to compute the partition function sums in (四), we take a constant level spacing from the highest available excited states to complete the partition sum as a geometric series. This method does not include the loosely-bound states $\nu=1,2,3 \ldots$, of which we estimate there are $\sim 10$ for each $m$, spread between $\sim 1 / 3$ the tight binding energy and the suppressed continuum. At the highest temperatures present in our atmospheres, the Boltzmann suppression for these states only partly compensates for their large number, and they may contribute significantly to the partition function. Under these conditions, more detailed treatments of ionization equilibrium will require exhaustive line lists, and a means of calculating pressure effects on partition sums and ionization tailored to the magnetic plasma.

Our equation of state under these extreme conditions is of necessity approximate, despite some justified simplifying assumptions. Although the gas deep in our atmospheres would be degenerate at low fields, with high $B$ the degeneracy is strongly suppressed. In particular, the gas is non-degenerate if $T \gg T_{F}=0.6\left(\rho\left(Z_{\text {eff }} / 26\right)\right)^{2} / B_{12}^{2}$ (Hernquist 1984). For our atmospheres, $B_{12}^{2}=10-100$ and $\rho\left(Z_{\text {eff }} / 26\right)<10^{3}$, so the gas is far from degeneracy throughout (Fig. 2). Also $k T \ll \hbar \omega_{B}$; in the adiabatic limit, this ensures that only one Landau level is occupied, although more exact treatments require the consideration of higher $n$ (Potehkin, Pavlov and Ventura 1997). The plasma parameter $\Gamma=Z_{\text {eff }}^{2} e^{2}\left(4 \pi n_{i} / 3\right)^{1 / 3} / k T$, where $n_{i}$ is the ion number density, is far below the solidification value $(\Gamma \sim 170)$. While our atmospheres do extend well past $\Gamma=1$ at high $T_{\text {eff }}$, we ignore the increasing non-ideality under these conditions (eg. Fehr and Kraft 1995). Accordingly, we solve for the ionization equilibrium conditions iteratively, using (1), to find the number density of all species (and hence $\rho(P, T)$ ) required by the ideal gas law to produce the pressure $P(\tau)$ needed for hydrostatic equilibrium ( $(6)$. The mean ionization level $\left\langle Z_{\text {eff }}\right\rangle$ is shown for selected $\tau$ in Fig. 2. We have tested the sensitivity of our final results to the omission of non-ideality corrections to the equation of state in two ways. First, we have adopted $20 \%$ of the Debye-Hückel correction as approximately appropriate for the densities near the base of our atmospheres (Rogers 1981), to estimate the structure perturbation in our $10^{6} \mathrm{~K}$ atmosphere model. Holding the temperature run fixed, we find that although there are $\sim 5 \%$ variations in density at the largest depths considered, at the surface the differences in the emergent spectrum are below $\sim 1 \%$ near the peak, and the total flux difference is $\sim 0.25 \%$. We have also made the extreme assumption that the plasma becomes an incompressible liquid at $\Gamma=3$ and tested the effect on the emergent spectrum: $\sim 5 \%$ changes were seen in the emergent flux near the weakly 
bound lines, but significant continuum variation was found only for $E_{\gamma}>20 k T_{\text {eff }}$. We therefore ignore plasma corrections in the following computations.

\section{Opacities}

\subsection{Radiation Propagation in the Magnetized Plasma}

In a magnetized plasma, radiation may propagate only in two specific polarization modes, called the normal modes. Their properties are obtained in general by finding the polarizability tensor of the plasma, and using it to solve Maxwell's equations for the case of a plane wave. For a collisionless magnetized plasma, the resulting mode polarization components are easily given (Ginzburg 1970) in the co-ordinate system $\left(x^{\prime}, y^{\prime}, z^{\prime}\right)$, where $z^{\prime}$ is in the propagation direction $\hat{k}$, and the magnetic field direction $\hat{B}$ is in the $y^{\prime}-z^{\prime}$ plane, at angle $\theta$ from the $z^{\prime}$ axis. The relative strengths of the components for modes $j=1,2$ are

$$
\begin{gathered}
\frac{e_{y^{\prime}}^{j}}{e_{x^{\prime}}^{j}}=-i \frac{2 u^{1 / 2}(1-v) \cos \theta}{u \sin ^{2} \theta+(-1)^{j-1}\left(u^{2} \sin ^{4} \theta+4 u(1-v)^{2} \cos ^{2} \theta\right)^{1 / 2}} \\
e_{z^{\prime}}^{j}=-i \frac{u^{1 / 2} v \sin \theta}{u-(1-v)-u v \cos ^{2} \theta} e_{x^{\prime}}^{j}+\frac{u v \cos \theta \sin \theta}{u-(1-v)-u v \cos ^{2} \theta} e_{y^{\prime}}^{j},
\end{gathered}
$$

where $u \equiv\left(\omega_{B} / \omega\right)^{2}$, and $v \equiv\left(\omega_{p} / \omega\right)^{2}$ with $\omega_{p}=4 \pi n_{e} e^{2} / m$ the plasma frequency.

We normalize these components, and rotate them to the basis $(x, y, z)$ with $z$ in the $\hat{B}$ direction, and $\hat{k}$ in the $y$ - $z$ plane, retaining the definition of $\theta$, not to be confused with the angle $\theta_{n}$ between $\hat{k}$ and the atmosphere's outward normal. Finally we construct $e_{+}^{j} \equiv\left(e_{x}^{j}+i e_{y}^{j}\right) / \sqrt{2}$ and $e_{-}^{j} \equiv\left(e_{x}^{j}-i e_{y}^{j}\right) / \sqrt{2}$, the polarization components in the rotating co-ordinate basis in which the opacities are most easily calculated. The indices of refraction are

$$
n_{j}^{2}=1-\frac{2 v(1-v)}{2(1-v)-u \sin ^{2} \theta+(-1)^{j}\left(u^{2} \sin ^{4} \theta+4 u(1-v)^{2} \cos ^{2} \theta\right)^{1 / 2}}
$$

For the large $u$ we consider, the $j=2$ mode has an imaginary index of refraction $(n=i \widetilde{n}, \widetilde{n}$ real $)$ below the plasma frequency, when $1<v<\sec ^{2} \theta$. In this case we add the extra "skin-depth"

opacity $\tau_{\delta}(\omega)=\widetilde{n} \omega / c$ to the total mode opacity of $\$ 4.4$. In practice all but a negligible fraction of the emergent flux from our atmospheres is above the plasma frequency at the depth of spectrum formation.

In fact, absorptive terms in the polarizability tensor corresponding to the various opacities affect the mode decomposition. As a test, we treat electron-ion collisions as in Ginzburg (1970), approximating non-magnetic free-free absorption: the effect is negligible except well below the plasma frequency. Nonetheless we expect that proper inclusion of the atomic opacities will change the mode polarizations somewhat, especially in the line regions. To date, the only treatment of this effect is for hydrogen (Bulik \& Pavlov 1996). 


\subsection{Thomson Scattering and Free-Free Absorption Opacities}

Meszaros (1992) gives the normal mode electron scattering and free-free opacities as

$$
\sigma=\left(\sigma_{T}, \sigma_{f f}\right)\left[\frac{\omega^{2}}{\left(\omega+\omega_{B}\right)^{2}}\left|e_{+}\right|^{2} g_{\perp}+\frac{\omega^{2}}{\left(\omega-\omega_{B}\right)^{2}}\left|e_{-}\right|^{2} g_{\perp}+\left|e_{z}\right|^{2} g_{\|}\right]
$$

For Thomson scattering, $\sigma_{T}=(8 \pi / 3) \alpha_{F}^{2}(\hbar / m c)^{2}$ is just the non-magnetic cross-section, where $\alpha_{F}$ is the fine structure constant, and $g \equiv 1$. For free-free absorption (Brehmsstrahlung),

$$
\sigma_{f f}=4 \pi^{2} \alpha_{F}^{3} \frac{\hbar^{2} c^{2}}{m}\left\langle Z_{\text {eff }}^{2}\right\rangle n_{i} \frac{1-\exp (-\hbar \omega / k T)}{\omega^{3}(\pi m k T / 2)^{1 / 2}}
$$

is also the field-free cross-section, and the Gaunt factors are given by

$$
\begin{aligned}
& g_{\perp}(\omega, T, B)=\frac{1}{2} \int_{-\infty}^{\infty} \exp \left[-p^{2} /(2 m k T)\right] \frac{C_{1}\left(a_{+}\right)+C_{1}\left(a_{-}\right)}{\left(p^{2}+2 m \hbar \omega\right)^{1 / 2}} \\
& g_{\|}(\omega, T, B)=\int_{-\infty}^{\infty} \exp \left[-p^{2} /(2 m k T)\right] \frac{a_{+} C_{0}\left(a_{+}\right)+a_{-} C_{0}\left(a_{-}\right)}{\left(p^{2}+2 m \hbar \omega\right)^{1 / 2}} \\
& a_{ \pm}=\left(p \pm\left[p^{2}+2 m \hbar \omega\right]^{1 / 2}\right)^{2}\left(2 m \hbar \omega_{c}\right)^{-1} \\
& C_{0}(a)=\exp (a) E_{2}(a) / a \quad C_{1}(a)=\exp (a)\left[E_{1}(a)-E_{2}(a)\right],
\end{aligned}
$$

(e.g. Meszaros 1992), where $E_{1}$ and $E_{2}$ are the exponential integral functions. Under the conditions of our atmospheres, these Gaunt factors are $\sim 1$ to 10 ; they slightly steepen the inverse dependence of opacity on frequency.

\subsection{Bound-Free and Bound-Bound Opacities; Line Broadening}

Taking the bound-free $(+,-, z)$ basis cross sections from the Hartree-Fock wave function sums, we add the contributions from all electrons in each ion. The result for any excitation state of an ion is a bound-free edge and a falloff with complex structure. In the case of hydrogen, these features can be affected by the thermal motion of the ion transverse to the field (Kopidakis, Ventura \& Herold, 1996); and, if adiabaticity is not assumed, by resonances with states with non-zero Landau level (Potekhin, Pavlov \& Ventura, 1997). We expect the former effect to be much diminished for iron, while the latter could be as significant as other inaccuracies inherent in the 'adiabatic approximation' (see $\oint_{2}$ ). When atoms are excited to a state for which an explicit bound-free cross section is not available, we assign them the cross section of the highest available state.

To estimate the line opacity we use a list of energy levels from the Hartree-Fock modeling. Because of uncertainty in the energy level computations, line energies may be in error by as much as $10 \%$, but the general distribution of bound-bound transitions is representative of the true spectrum. We focus here on dipole allowed transitions, which obey the selection rules 
$\Delta m= \pm 1$ for even $\Delta \nu$, and $\Delta m=0$ for odd $\Delta \nu$ (Ruder et al. 1994). We find that in the adiabatic approximation, the energy level spectra for individual ions are roughly self-similar. Therefore, for each ionization state we use the $\Delta m=0, \Delta \nu=1$ transition of the outermost ground state electron, which we always have, to (linearly) re-scale the most extensive line energy lists available (for $Z_{\text {eff }}=2$ at the lower field, $Z_{\text {eff }}=0$ at the higher) and apply it to the ion. For most ions we have explicitly computed a few allowed transition energies; these are always used, but in any case they match well to the predicted energy from the re-scaled line lists. We have attempted to compute a 'complete' set of dipole allowed transitions from the ground states only; we generally do not have explicit energies for all relevant bb transitions from excited states. To approximate the total distribution of oscillator strength from these transitions, we estimate the number of missing lines for each excited state and assign the ground-state line spectrum to the unaccounted-for fraction. While we have not computed the oscillator strengths for all transitions in detail, we can estimate the line opacity by noting that the strengths, defined by $\int \sigma(E) \mathrm{d} E=\left(2 \pi^{2} e^{2} \hbar / m c\right) f_{\text {osc }}$, take on characteristic sizes for the different transitions. Furthermore, the type of transition determines to which component of the polarization basis the line couples. We use $f_{\text {osc }}($ couples to + mode $) \approx 0.06(\Delta m=1, \Delta \nu=0) ; f_{\text {osc }}(z$ mode $) \approx 0.8,0.06$ $(\Delta m=0, \Delta \nu=1,3)$; and $f_{\mathrm{osc}}(+,-) \approx 5 \times 10^{-4}, 5 \times 10^{-5}(\Delta m=1,-1 ; \Delta \nu=2)$ for the oscillator strengths at $B=10^{12.5} \mathrm{G}$. For $B=10^{13} \mathrm{G}$, the corresponding strengths are $f_{\text {osc }}(+) \approx 0.04$ $(\Delta m=1, \Delta \nu=0) ; f_{\text {osc }}(z) \approx 0.4,0.03(\Delta m=0, \Delta \nu=1,3) ;$ and $f_{\text {osc }}(+,-) \approx 9 \times 10^{-5}, 9 \times 10^{-6}$ $(\Delta m=1,-1 ; \Delta \nu=2)$. These oscillator strengths are accurate to within a factor of $\sim 2$, which is sufficient for our modeling needs.

We include two very different broadening mechanisms in our calculation. First, at the high densities and temperatures characteristic of neutron star atmospheres, line features experience significant collisional broadening. For an electron in an orbital of characteristic radius $a$ about a nucleus of charge $Z$, the energy shift due to the dipole induced by an external electric field $F$ is $\Delta E \sim F^{2} a^{3} / Z$. For collisional impact, we have $F=e / r_{\text {impact }}^{2}$, and $Z=Z_{\text {line }}$ the net charge interior to the electron absorbing the photon. We use the Weisskopf method to approximate the broadening, since the impinging electrons are confined by the magnetic field to move in a straight line, giving a Lorentzian cross-section with width

$$
\hbar \Gamma_{\text {coll }}=\pi^{5 / 3} 2^{1 / 3} \hbar^{1 / 3} a^{2} e^{4 / 3}\left(\frac{k T}{m_{e}}\right)^{1 / 6} \frac{n_{e}}{Z_{\text {line }}^{2 / 3}} \sim 41.5 T_{6}^{1 / 6} \frac{\left(n_{e} / 10^{24}\right)}{Z_{\text {line }}^{8 / 3}}\left[\frac{a}{\left(a_{0} / Z_{\text {line }}\right)}\right]^{2} \mathrm{eV},
$$

which comes from the amplitude and $r$-dependence of $\Delta E$ (see Mihalas 1978). For $a$ we use the largest dimension of initial or final state, with dimension $a_{\perp}$ transverse to the field as given in $\S$, and $a_{z}$ parallel to the field estimated as follows. Orbitals with $\nu=0$ are localized near the nucleus; we need their $a_{z}$ only if it is bigger than $a_{\perp}$, in which case it is given, through minimizing the total energy of an annular charge distribution, by $a_{z}=a_{0} / \ln \left(a_{0} / a_{\perp}\right)$ (e.g. Meszaros $\left.1992 \S 2.4\right)$. For $\nu>0$ we estimate $a_{z}=\left(a_{0} / Z_{\text {line }}\right)[(\nu+1) / 2]^{2}$, which comes analytically from the Schrödinger equation for $f_{0 m \nu}(\S 2)$ for odd $\nu$ states of hydrogen at high field (Ruder et al. 1994, §6.4.4). Since energies vary smoothly with $\nu$ regardless of parity, both in that work and in ours, we use the 
above estimate for even $\nu$ also.

Secondly, the thermal motion of the ions past the large $B$ also causes significant energy level perturbations (Pavlov \& Meszaros 1993). We can approximate this effect by a Lorentz electric field $F=\left(v_{\perp} / c\right) B$, where $v_{\perp}$ is the velocity transverse to the magnetic field. As in the case of hydrogen, we take this 'magnetic broadening' to be one-sided, since the Lorentz field always results in elongation of the otherwise circular transverse wave function. We estimate the energy shift as above by $\Delta E \sim F^{2} a^{3} / Z_{\text {line }}$, taking $a$ to be the larger $a_{\perp}$ of initial and final state, since $F$ must be transverse to $B$. Using $v_{\perp} \sim \sqrt{2 k T / m_{\mathrm{Fe}}}$ we get $F=5.8 \times 10^{7} B_{12} \sqrt{T_{6}} \mathrm{cgs}$, yielding a width of

$$
\hbar \Gamma_{\text {mag }}=0.035(2 m+1)^{3 / 2} \frac{T_{6} \sqrt{B_{12}}}{Z_{\text {line }}} \mathrm{eV} .
$$

Making proper allowance for the higher thermal velocity of hydrogen, equation (8) agrees with the numerical example in Pavlov \& Meszaros (1993) within a factor of 2. Note that since it is independent of density, magnetic broadening is most important near the surface of our atmospheres. We add the two broadening mechanisms, giving a redward half-Lorentzian with width $\hbar \Gamma_{\mathrm{R}}=\hbar \Gamma_{\text {mag }}+\hbar \Gamma_{\text {coll }}$, and a blue line wing with width $\hbar \Gamma_{\mathrm{B}}=\hbar \Gamma_{\text {coll }}$. The line profile, normalized to the $f_{\text {osc }}$ as above, is thus

$$
\begin{array}{cc}
\sigma(E) \mathrm{d} E=\frac{\pi e^{2} \hbar f_{\text {osc }}}{m c}\left(\frac{2 \Gamma_{\mathrm{R}}}{\Gamma_{\mathrm{R}}+\Gamma_{\mathrm{B}}}\right)\left(\frac{\hbar \Gamma_{\mathrm{R}}}{\left(E-E_{0}\right)^{2}+\left(\hbar \Gamma_{\mathrm{R}} / 2\right)^{2}}\right) \mathrm{d} E & E \leq E_{0} \\
\sigma(E) \mathrm{d} E=\frac{\pi e^{2} \hbar f_{\mathrm{osc}}}{m c}\left(\frac{2 \Gamma_{\mathrm{R}}}{\Gamma_{\mathrm{R}}+\Gamma_{\mathrm{B}}}\right)\left(\frac{\left(\Gamma_{\mathrm{B}} / \Gamma_{\mathrm{R}}\right) \hbar \Gamma_{\mathrm{B}}}{\left(E-E_{0}\right)^{2}+\left(\hbar \Gamma_{\mathrm{B}} / 2\right)^{2}}\right) \mathrm{d} E & E \geq E_{0},
\end{array}
$$

where $E_{0}$ is the line energy. As this net broadening is sufficient to blend many of the lines, the exact values of the line energies are not crucial in estimating the b-b contribution to the overall opacity.

\subsection{Total Radiative Opacity}

When our atmosphere calculation calls for the radiative opacity in each mode at a given angle and frequency, for some ambient temperature and density, we sum the scattering, free-free, bound-free and bound-bound $(+,-, z)$ cross sections for that frequency over all ionization and excitation states weighted by (1) at the appropriate temperature and density, giving $\sigma_{+,-, z}(\omega, \theta, T, \rho)$. The mode cross-sections are then given by

$$
\sigma_{j}(\omega, \theta, T, \rho)=\sum_{i=+,-, z} e_{i}^{j}(\omega, \theta)^{2} \sigma_{i}(\omega, T, \rho) \quad j=1,2
$$

We convert these from $\mathrm{cm}^{2} /$ particle to $\mathrm{cm}^{2} / \mathrm{g}$, and multiply by the density to get the opacity $\kappa_{j}(\omega, \theta, T, \rho)$ in $\mathrm{cm}^{-1}$. 


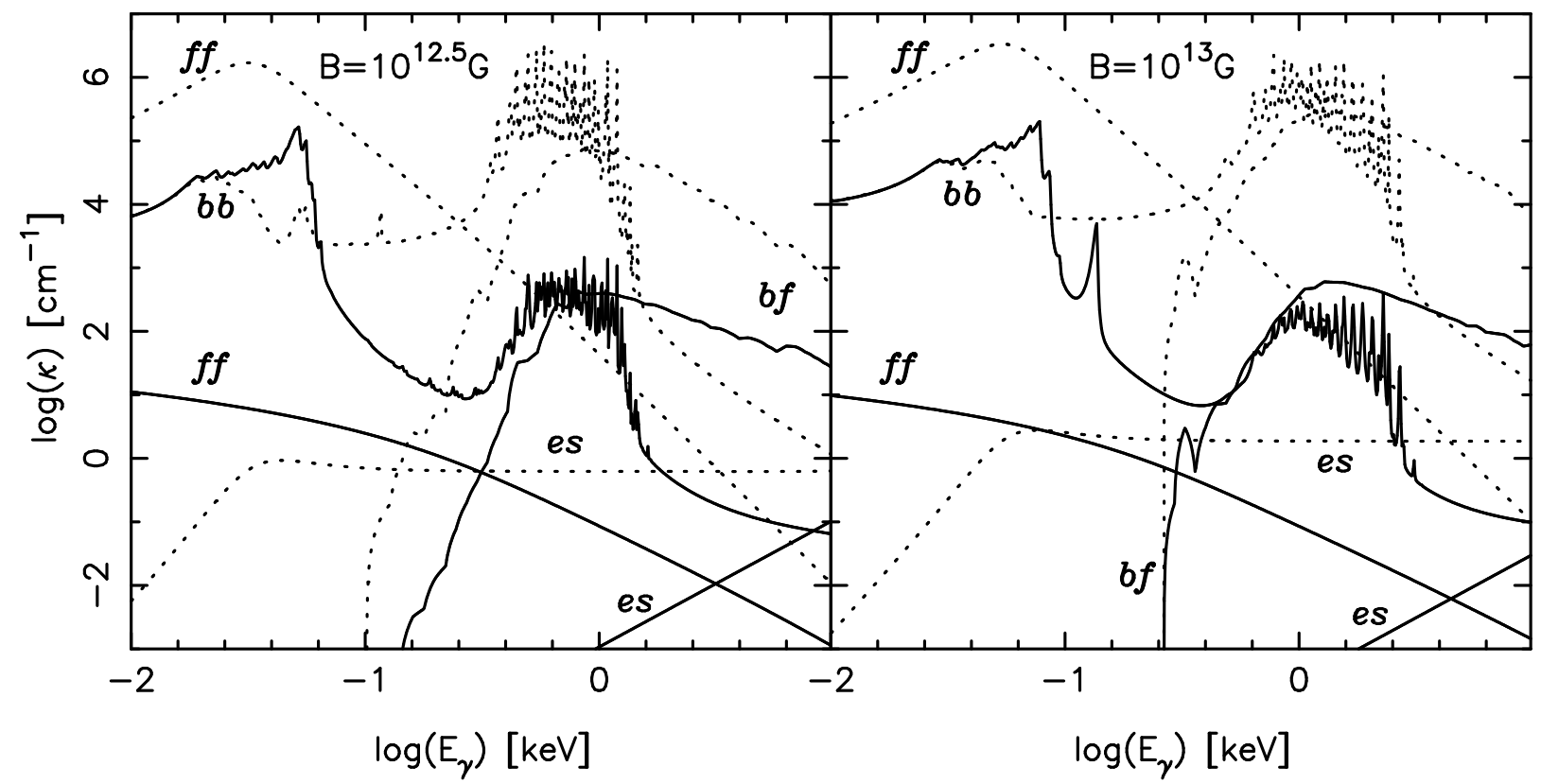

Fig. 1.- Left: Contributions to the radiative opacity at $\theta=55^{\circ}$, at total mean optical depth (defined in $\AA_{6}$ ) $\tau_{T}=1$ in our $B=10^{12.5} \mathrm{G}, T_{\text {eff }}=10^{6} \mathrm{~K}, \Theta_{B}=0^{\circ} \mathrm{Fe}$ atmosphere. The two modes are shown by solid and dashed lines respectively. Note that significant b-f opacity is present at large $E$. b-b transitions between tightly bound states appear at low energy; tight-weak transitions are at higher energy. Right: Same for $B=10^{13} \mathrm{G}$.

To illustrate the relative importance of different contributions to the opacity, we show opacity spectra for the two normal modes at $55^{\circ}$ to the magnetic field, under characteristic atmosphere conditions. Free-free opacity dominates at low energies, but b-f and b-b processes contribute significant opacity at higher energies; electron scattering is never significant in these Fe atmospheres.

\section{Energy Transfer in the Atmosphere}

\subsection{Radiative Transfer}

The magnetic field, at an angle $\Theta_{B}$ to the atmosphere normal, causes significant anisotropies in the energy transfer. In addition, ZPS96 have shown that even without a field there can be substantial limb darkening and that the emergent spectrum will vary with $\mu=\cos \theta_{n}$ the $\operatorname{cosine}$ of the viewing angle to the atmosphere normal. For emission from a small region on the star, these effects will cause substantial variation with the viewing angle. We therefore follow the angular dependence of both internal radiation field and emergent spectrum, although our atmosphere 
temperature structure remains plane parallel.

We define a grid of angular co-ordinates $(\theta, \phi)$ about the magnetic field direction. Given the state of the atmosphere at each depth, we use the opacities of $\S 3$ to compute frequencyand $\theta$ - dependent optical depths, by summing $\tau_{j}(d, \omega, \theta)=\int \kappa_{j}(\omega, \theta, T, \rho) \mathrm{d} z$ from the surface to atmosphere layer number $d$, for each theta in our grid. This precedes any consideration of the atmosphere normal direction, and the sums are done using the normal thickness $\mathrm{d} z$ of each layer.

We next compute radiation fields at each $\theta$ and $\phi$ at a given atmosphere level. The intensity of outward going radiation is given by

$$
I_{j}\left[\tau_{j}(\omega, \theta), \mu\right]=\int_{\tau_{j}}^{\infty}\left[B_{\omega}\left(\tau^{\prime}\right) / 2\right] e^{-\left(\tau^{\prime}-\tau_{j}\right) / \mu} \mathrm{d} \tau^{\prime} / \mu \quad(0 \leq \mu \leq 1)
$$

and inward going radiation by

$$
I_{j}\left[\tau_{j}(\omega, \theta), \mu\right]=\int_{0}^{\tau_{j}}\left[B_{\omega}\left(\tau^{\prime}\right) / 2\right] e^{-\left(\tau_{j}-\tau^{\prime}\right) /(-\mu)} \mathrm{d} \tau^{\prime} /(-\mu) \quad(-1 \leq \mu \leq 0)
$$

where $\mu=\cos \theta \cos \Theta_{B}+\sin \theta \sin \Theta_{B} \cos \phi$ (taking the normal direction to have $\phi=0$ by definition) and $B_{\omega}(T)=\left(\hbar \omega^{3} /\left[2 \pi^{2} c^{2}\left(e^{\hbar \omega / k T}-1\right)\right]\right.$ is the Planck function, half of which gives the source function in each mode for LTE. When $\Delta \tau_{j}(\omega, \theta)>20$ in a given layer we use the diffusion approximation for that frequency and mode: $I\left[\tau_{j}(\omega, \theta), \mu\right]=B_{\omega}\left[\tau_{j}(\omega, \theta)\right]+\mu \partial B_{\omega} / \partial \tau_{j}(\omega, \theta)$. The intensities are then summed to find the astrophysical flux $F_{j \omega}=(1 / \pi) \iint \mu I_{j}(\omega, \theta, \phi) \mathrm{d} \Omega$. We find that 18 zones in $\theta$ and 9 zones in $\phi$ (zero to $\pi$ only, by symmetry) give better than $0.1 \%$ accuracy in the intensity integral even for test functions with rapid variations in $\theta$ and $\phi$. To monitor atmosphere flux the above computations are performed on 200 energy bins, logarithmically spaced between $1 \mathrm{eV}$ and $10 \mathrm{keV}$.

\subsection{Electron Thermal Conductivity}

Electron thermal conduction provides a significant component of the heat flux at large depth in our coolest atmosphere models. The electron thermal conductivity is significantly modified under high field conditions (Hernquist 1985). Because the electron gas in our atmospheres is non-degenerate (see $\S$ [), and occupies only the lowest Landau level, the conductivity $\lambda_{\|}$ (Hernquist's $\kappa_{\|}$) along $\hat{z}$ can be readily computed by evaluating the integrals in Hernquist's Eq (3.5), using (3.8) and (3.10). The chemical potential is given by (2.12), and its derivative required by $(3.10 \mathrm{c})$ is taken analytically. Electron conduction perpendicular to $B$ will be much smaller, and we take it to be zero. We find that in our coldest $B=10^{13} \mathrm{G}$ atmosphere, in which it is most significant, the electron conductivity carries up to $80 \%$ of the heat flux at $\tau_{\text {rad }}=10$ (defined below). The extra mode of transport affects the temperature structures and emergent spectra significantly. 


\section{Converging the Atmosphere and Obtaining Spectra}

Deep within the atmosphere, the optical depths are large for all frequency bins, and radiation flows in the diffusion limit with flux $F_{\omega}=(1 / \pi) \iint \mu(\theta, \phi)^{2}\left(\mathrm{~d} B_{\omega} / \mathrm{d} \tau_{\omega} \theta\right) \sin \theta \mathrm{d} \theta \mathrm{d} \phi$. We therefore define a radiative mean opacity

$$
\kappa_{\text {rad }}^{-1}=\frac{\pi}{4 \sigma T^{3}}\left[\int_{0}^{\infty} \frac{\partial B_{\omega}}{\partial T} \frac{3}{4 \pi}\left(\int_{0}^{2 \pi} \int_{0}^{\pi} \frac{1}{2} \sum_{j} \frac{\mu(\theta, \phi)^{2}}{\kappa_{j}(\omega, \theta)} \sin \theta \mathrm{d} \theta \mathrm{d} \phi\right) \mathrm{d} \omega\right]
$$

equivalent to the Rosseland mean opacity; the corresponding optical depth scale is $\tau \mathrm{rad}$. The

total mean opacity is then given by $\kappa_{\mathrm{T}}^{-1}=\kappa_{\mathrm{rad}}^{-1}+\kappa_{c}^{-1}$, where $\kappa_{c}=16 \sigma T^{3} /\left(3 \lambda_{\|} \cos \Theta_{B}\right)$ gives the effective conductive contribution. This total opacity defines a useful depth scale $\tau_{\mathrm{T}}<\tau \mathrm{rad}$, as the temperature structure tends to the grey solution at large depth on this scale. Our atmospheres have 146 depth zones with $-6<\log \tau_{\mathrm{T}}<2$; the very low $\tau$ layers are required by the extreme frequency and angle dependence of our opacities.

As usual we relate the optical depth zones to physical depths by summing the equation of hydrostatic equilibrium down from the surface: $\mathrm{d} P=\left[g_{s} \rho / \kappa_{\mathrm{T}}\left(\tau_{\mathrm{T}}\right)\right] \mathrm{d} \tau_{\mathrm{T}}$. Because $\kappa_{T}=\kappa_{T}(\rho, T)$, we solve iteratively for the physical zone size ( $c f$. RR96). We then evaluate the total radiative flux $F_{\text {rad }}=\sum_{j=1,2} \int F_{j \omega} \mathrm{d} \omega$ as above at each depth zone, and add the conductive heat flow $F_{c}=\left(\lambda_{\|} / \pi\right) \mathrm{d} T / \mathrm{d} z$ to obtain the total energy flux at each depth. This is adjusted to the constant flux solution, using the Lucy-Unsöld temperature correction scheme, as in RR96. We iterate until flux errors are $<1 \%$ throughout the atmospheres.

In the surface layers, we also consider the behavior of $f=\int \mathrm{d} \omega \int \sin \theta \mathrm{d} \theta \mathrm{d} \phi \sum_{j=1}^{2} \kappa_{j}\left(I-B_{\omega} / 2\right)$. Although convergence to $f=0$ is a useful diagnostic, we find that a simple $\Lambda$ iteration scheme using $f$, as suggested by Shibanov et al. (1995), provides no help in reaching convergence. Full scale Feautrier schemes could, however, speed convergence considerably.

As noted above, both angle dependent specific angular fluxes and the total emergent spectrum are of interest. These are computed from equation (12) at the surface layer at $10^{3} \log$-spaced energies for higher spectral resolution.

\section{Results and Conclusions}

We converge atmospheres with effective temperatures $T_{\text {eff }}=10^{5.5}, 10^{6.0}$ and $10^{6.5} \mathrm{~K}$, with vertical $\left(\Theta_{B}=0\right)$ magnetic fields of $10^{12.5}$ and $10^{13}$ Gauss. To examine the dependences on magnetic geometry, we also converge atmospheres with $\Theta_{B}=45^{\circ}$ and $90^{\circ}$ at $10^{6.0} \mathrm{~K}$ for both field strengths.

Figure 2 gives the temperature structure computed for the $\Theta_{B}=0$ atmospheres. Open circles show the locations of the mean radiative optical depths 1 and 10, labeled with the average ionization level $\left\langle Z_{\text {eff }}\right\rangle$ at these points. The temperature structure may be compared with 


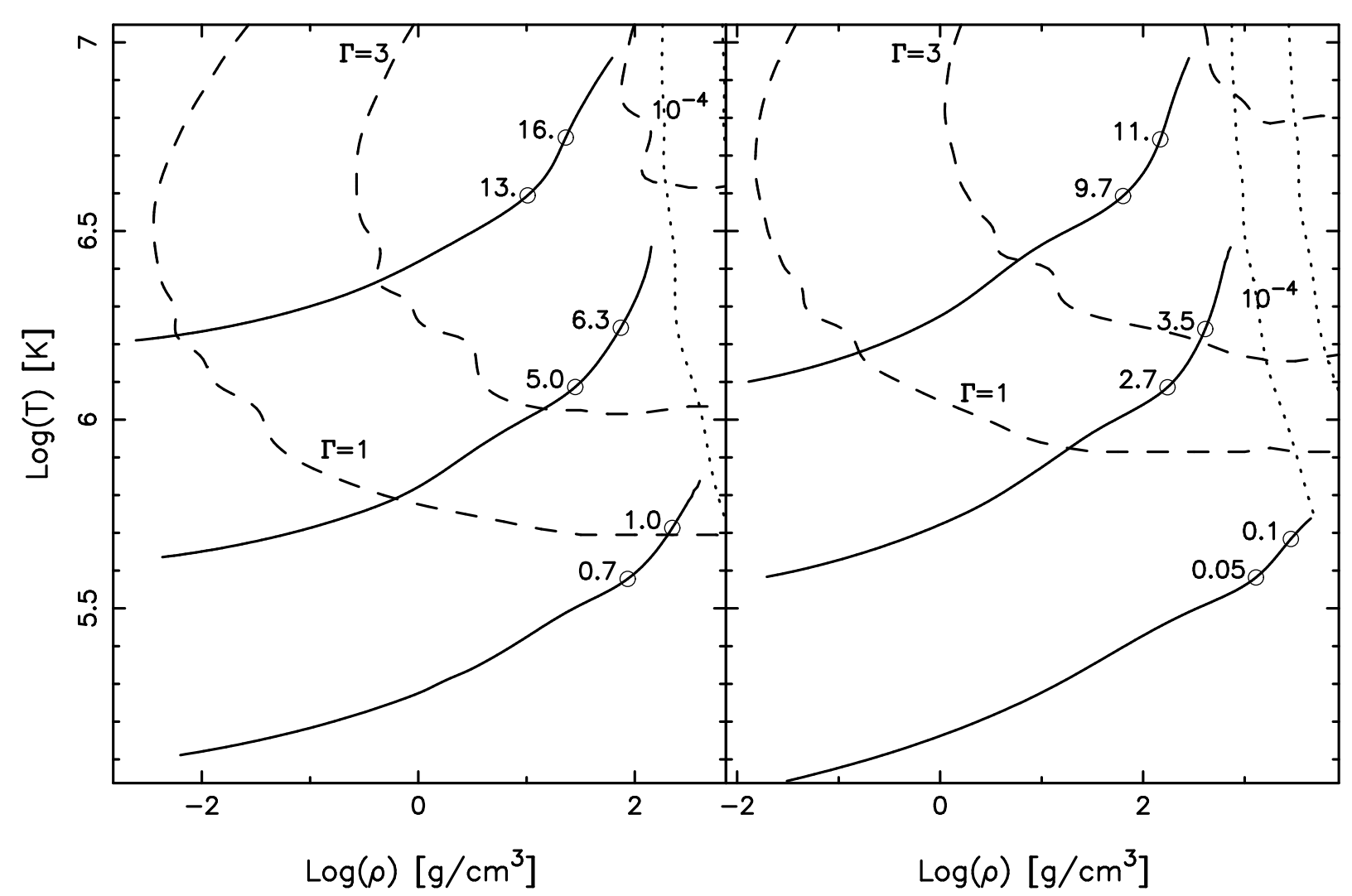

Fig. 2.- Left: $B=10^{12.5} \mathrm{G}, \Theta_{B}=0$ atmosphere structures $(\rho-T$ plane $)$ for $\log \left(T_{\text {eff }}\right)=5.5,6.0,6.5$ superimposed on curves for $E_{F} / k T=10^{-4}, 10^{-3}$ and $\Gamma=1,3$ and 10 curves. Positions of $\tau_{\text {rad }}=1$ and 10 (see $\S$ (6) are marked with open circles. Labels by the points give the mean ionization level at these depths. Right: Same for $B=10^{13} \mathrm{G}$.

background curves showing $\Gamma$ and $E_{F} / k T$; the depth zones dominating the spectrum lie in the gaseous non-degenerate regime.

Figure 3 shows the emergent spectra for the normal field atmospheres - these can be compared with the corresponding blackbodies. As in Romani (1987), RR96 and ZPS96 we see that the rich absorption spectrum of the heavy element gives an overall opacity trend much flatter than the steep Kramer's law fall-off of $\mathrm{H}$ and He. This assures that the spectra are globally closer to a blackbody shape than the light element atmospheres. In fact, the bound-free edges at $\sim 0.3-1.0 \mathrm{keV}$ provide a large increase in opacity that gives Fe spectral deficits above the Wien peak. Qualitatively, this is similar to the effect of L-edge absorptions in non-magnetic iron (RR96). Looking at the opacities and at the absorption features in the spectra, one can see that the magnetic Fe transitions are divided, roughly, into two energy ranges: $\Delta \nu=0$ transitions below $\sim 100 \mathrm{eV}$ (for our lower field) and all other transitions, mostly at energies above $300 \mathrm{eV}$. Interestingly, for typical neutron star magnetic fields these transitions are at energies roughly 
comparable to the M- and L-shell transitions in non-magnetic Fe. The resulting spectral shapes therefore have some similarities to non-magnetic iron. Note that we do not expect the detailed line positions in our atmospheres to be accurate; the general appearance of these emergent spectra should however be similar to more accurate results. Although more complete atomic data should give an even richer line spectrum, we do find that certain species dominate at depths where the lines are forming. Thus high resolution X-ray spectra can in principal extract useful information about the Fe atmosphere structure. In particular, the rather strong line broadening, most visible at low E, does not prevent discrete lines from being visible at higher energies. At low energies lines are also visible, but equivalent widths are questionable because of uncertainty in the detailed temperature structure in the very low $\tau$ surface layers.

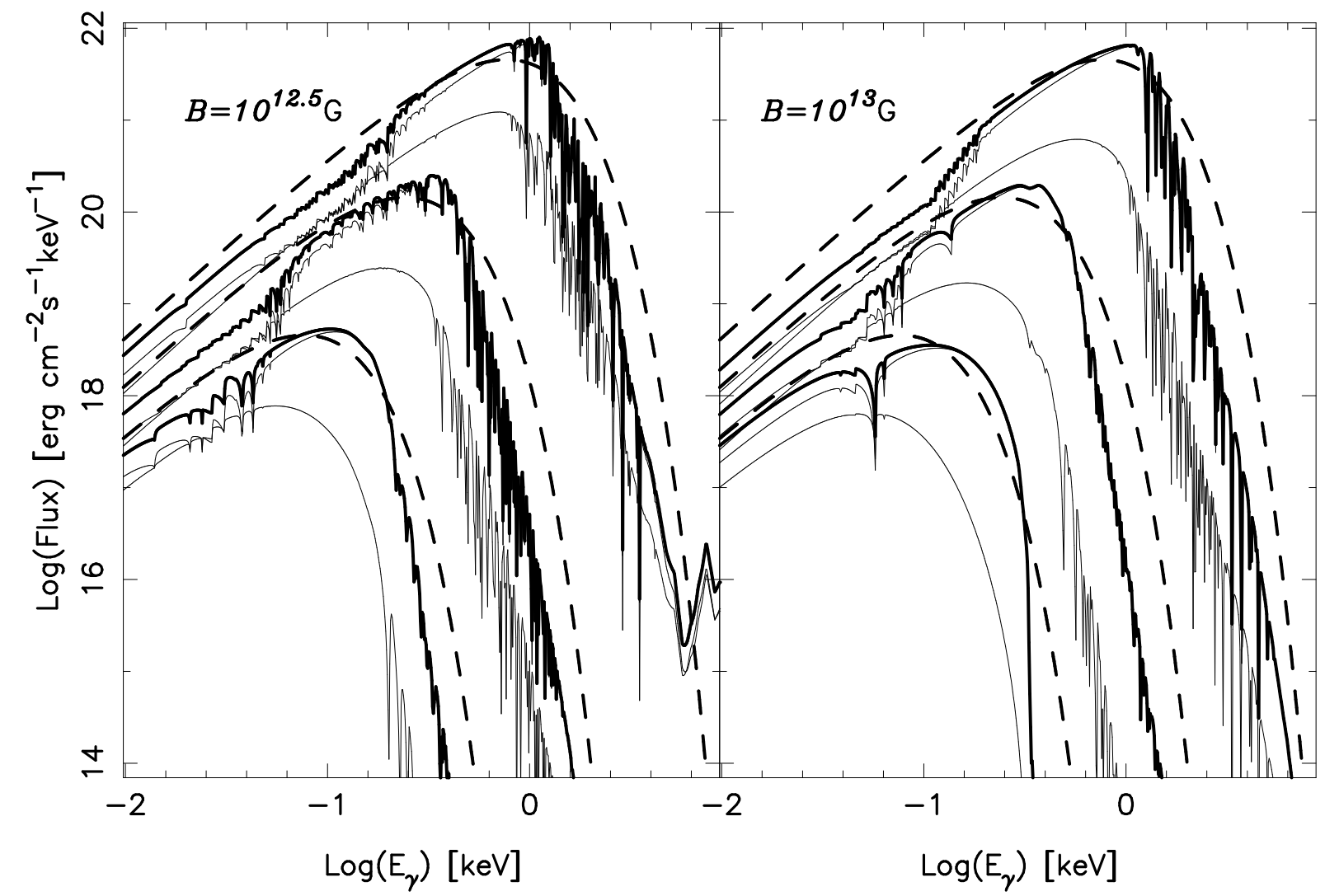

Fig. 3.- Left: Emergent Fe spectra for $B=10^{12.5} \mathrm{G}$ and $\Theta_{B}=0$ at $\log \left(T_{\text {eff }}\right)=5.5,6.0$ and 6.5 , showing the two modes (thin solid lines), the total flux (heavy solid line) and corresponding black-body spectrum (dashed). Right: Emergent spectra for $B=10^{13} \mathrm{G}$.

In Figure 3 we also show the contributions of the two normal modes to the emergent flux at each temperature. While we do not discuss it in detail here, it is clear that the thermal emission will show a strong energy dependent polarization. This polarization also depends on the magnetic field geometry; thermal polarization from neutron star surfaces should be pulsed at the star's spin period. 
In practice, we expect quite substantial variation of the emergent spectrum over the surface. If due to a coherent dipole, the variation of magnetic field strength and orientation will affect the bulk flow of thermal flux from the interior to the star surface. For example Shibanov and Yakovlev (1996) indicate an effective temperature decrease as large as $\sim 7$ from the magnetic pole to the magnetic equator for a $10^{13} \mathrm{G},\left\langle T_{\text {eff }}\right\rangle=10^{6} \mathrm{~K}$ neutron star. Such temperature dependence implies large thermal emissivity variation with viewing angle. For a given $T_{\text {eff }}$ we find that the broadband spectral dependence on $\Theta_{B}$ is quite modest (see also Pavlov, et al. 1995). However, for a surface with varying $T_{\text {eff }}$, including the spectral dependence imposed by an atmosphere (e.g. as modeled for Geminga by Meyer, Pavlov and Meszaros 1994) can give a complex, energy dependent pulse behavior. In fact, spectral features are quite dependent on the magnetic field strength (Figure 3). At a minimum, even if the large scale dipole dominates the field at the surface, there will be a factor of two difference in $B$ between the magnetic equator and the magnetic poles. Higher order multipole contributions are also possible; the large field variations will blur any spectral features significantly.

Finally in addition to the magnetic field variation and effective temperature variation, there will be a significant modulation of the neutron star thermal spectrum with changes of the observer's viewing angle to an emitting region on the stellar surface. ZPS96 have emphasized the 'limb darkening' decrease in the emitted flux with increasing $\mu$ for non-magnetic atmospheres. In magnetized atmospheres the $\mu$ dependence can be extremely complicated in general. One basic effect is visible below $E_{\gamma}=0.1 \mathrm{keV}$ in the normal field case of Figure 4; here one of the two normal modes has a significant $z$ polarization component, which experiences a much higher continuum opacity unsuppressed by the field. The opacity increase acts in concert with the usual limb darkening to diminish the flux at small $\mu$. For tangential $\Theta_{B}=90^{\circ}$, on the other hand, when $\mu \sim 0$ in the $\hat{z}-\hat{n}$ plane both normal modes are primarily $(+,-)$, resulting in strongly suppressed continuum opacity. The extra transparency counteracts the limb darkening to some extent; the emissivity varies little below $0.1 \mathrm{keV}$ in the right panel of Figure 4 . Thus a simple dipole field neutron star would have a relatively bright limb at low $E_{\gamma}$ when viewed along the magnetic axis.

However, other effects can complicate this simplest picture. First, the lines and line wings can couple selectively to a single polarization component, and block out the modes transparent to the continuum. Secondly, when $\mu \sim 1$, the normal modes are circularly polarized and similar to one another, both containing small amounts of the $z$ component; while for $\mu \lesssim 0.7$ the modes are linearly polarized, and the mode perpendicular to the $B-\hat{k}$ plane is almost completely $z$-free. Thus when the $z$ opacity excess is too extreme, the $z$-free mode can have larger intensity at small $\mu$ than both modes together at $\mu \sim 1$. This effect is visible above $0.2 \mathrm{keV}$ in Fig. 4, where strong line wings are coupled to the $z$ component. In some cases even the continuum-controlled thermal peaks are dominated by this effect; however, any small change in normal mode composition can modify this behavior. Clearly models of purely thermal pulsed emission from neutron stars can be complex, with effective temperature, line feature position and limb darkening behavior controlled by the local magnetic geometry. These effects may be somewhat blurred by gravitational 


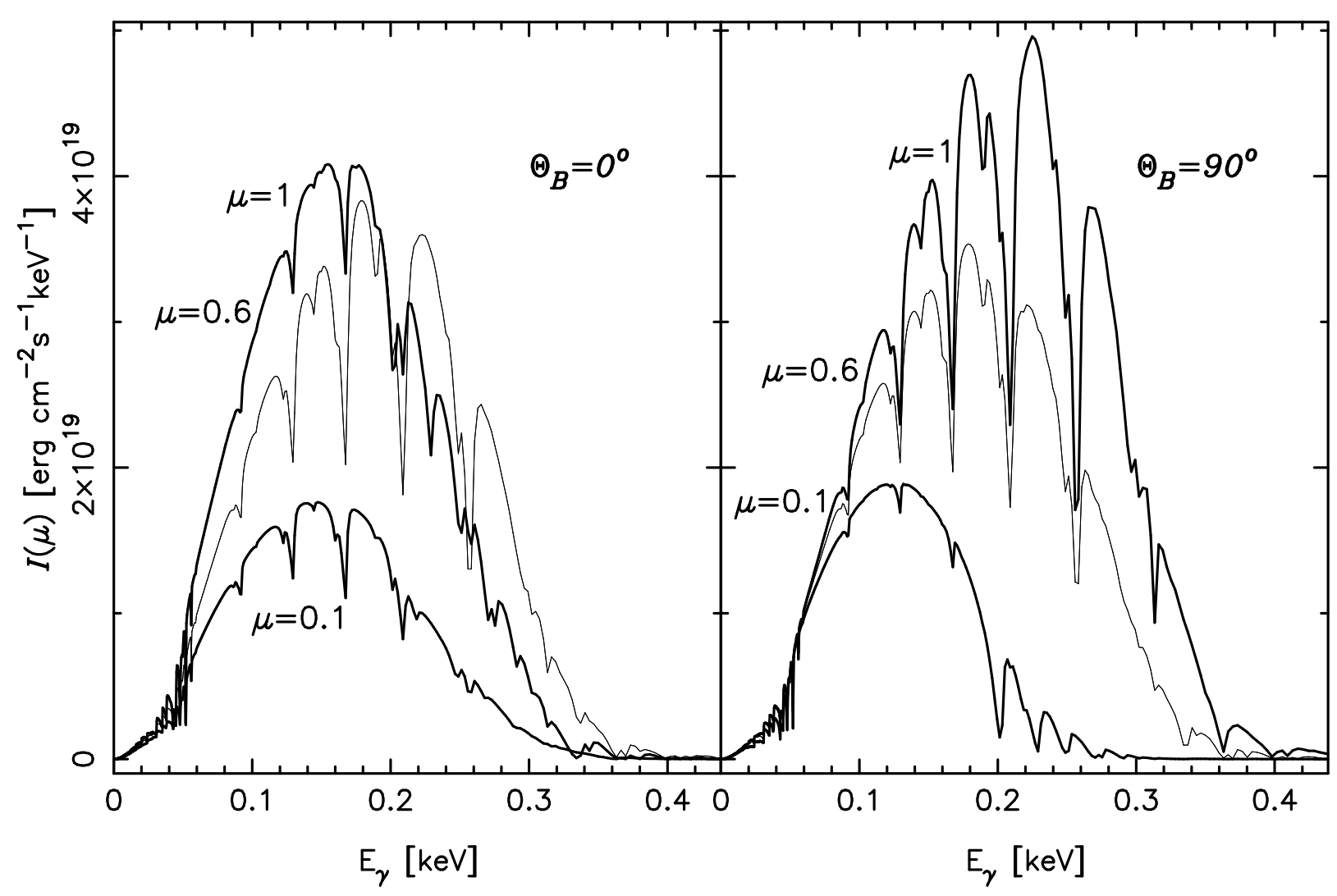

Fig. 4.- Left: Emergent Fe atmosphere emissivity as a function of viewing angle for $B=10^{12.5} \mathrm{G}$, $T=10^{5.75} \mathrm{~K}, \Theta_{B}=0$. Total fluxes are shown at $\mu=0.1,0.6$ (lighter curve) and 1.0. Note that the limb darkening is a strong function of energy. Right: Viewing angle dependence for $\Theta_{B}=\pi / 2$. The limb darkening, spectral features and polarization properties are a strong function of magnetic geometry.

defocusing of the emergent radiation (Page 1995), but substantial variation in the atmosphere features and overall emissivity are expected as the star rotates.

Our models suggest that if pulsars possess iron atmospheres, upcoming high throughput, moderate resolution spectroscopy missions such as XMM and AXAF will have a wealth of spectral features for diagnosis of atmosphere temperature structure and magnetic geometry. Data providing such rich spectra will certainly motivate more complete magnetic Fe models. Improvement of the atomic data sets used to generate the opacities will be essential for such models. Even exhaustive lists of configurations and lines, though, will have to be coupled with a more sophisticated treatment of the non-ideal equation of state, along the lines of an activity expansion of the grand canonical ensemble (Rogers 1986), or an occupation probability formalism (Hummer \& Mihalas 1988). Both these approaches, which have been used for non-magnetic plasma opacity calculations by the OPAL and OP collaborations respectively, avoid the somewhat 
ad hoc cutoff procedures necessary in using equation (1]). A fully consistent opacity/EOS system will also need to include the effects of plasma non-ideality on atomic (b-f and b-b) opacity. This will require calculation of states and energies of non-isolated atoms, desirable but prohibitively difficult at present. For the line opacities, the dipole selection rules and simple coupling of each line to a single polarization component are known to be a first approximation only, subject to complication by all forms of broadening. Presumably more detailed atomic modeling will address these topics, and produce more precise oscillator strengths as well. Finally, more realistic magnetic geometries and inclusion of (weak) mode coupling through scattering will have some effect on the emergent flux. Nonetheless, we expect our magnetic models have captured the qualitative behavior of heavy element atmospheres. In simplest terms this is that the wealth of spectral features bring the overall spectrum shape closer to a blackbody than for light element surfaces.

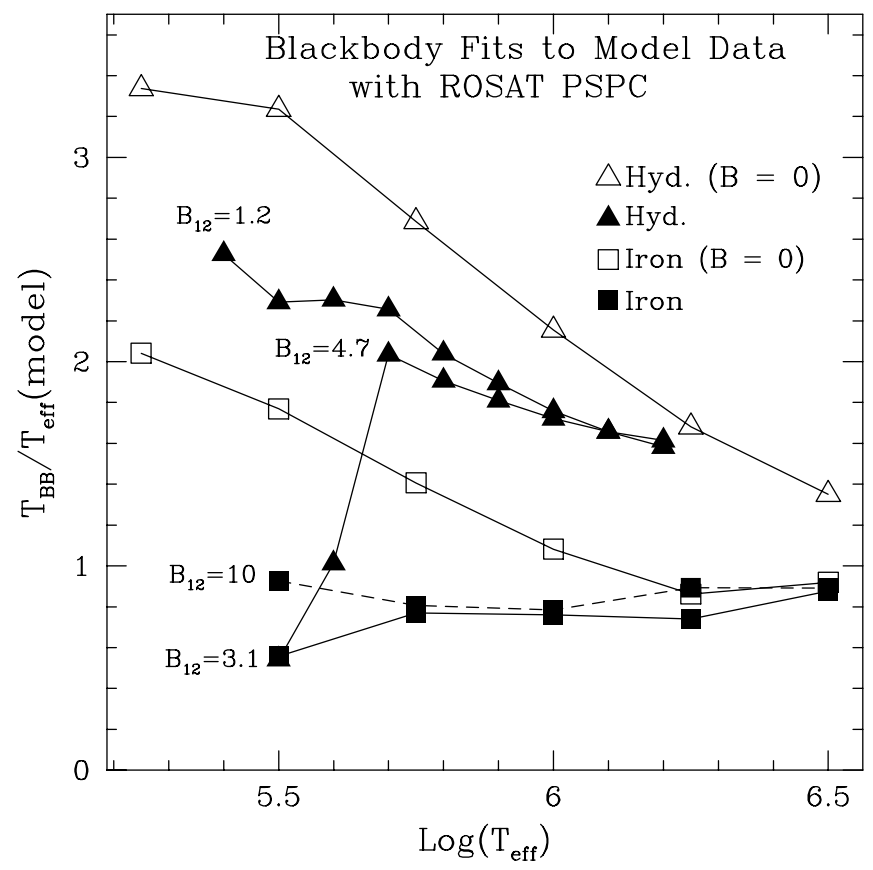

Fig. 5.- Inferred $T_{\text {eff }}$ when fitting a blackbody to a simulated 3000 count ROSAT PSPC spectrum produced by a redshifted $(z=0.306)$, absorbed $\left(N_{H}=10^{20} \mathrm{~cm}^{-2}\right)$ model atmosphere. Curves show the inferred $T$ for magnetic and non-magnetic $\mathrm{H}$ and $\mathrm{Fe}$ as a function of the true $T_{\text {eff }}$.

As in RR96, we can illustrate these broad band spectral effects by simulating a modest statistic (3000 count) PSPC exposure of a neutron star, whose absorbed spectrum is fitted by a blackbody. Comparing the inferred $T_{B B}$ with the true $T_{\text {eff }}$ for various atmosphere models illustrates the low resolution spectral effects (Figure 5). As for non-magnetic atmospheres, the Fe results are much softer for a given $T_{\text {eff }}$ than $\mathrm{H}$ atmospheres. In contrast, existing data suggest phase average spectra appreciably harder than the corresponding blackbody. While the clear existence of magnetospheric flux contributions and the likely $T_{\text {eff }}$ variations over the surface 
complicate the interpretation, it would seem that these data support the existence of light element atmospheres on neutron star surfaces. If substantiated, this conclusion would give important clues to the history of the neutron star surface and serious implications for cooling curves and the equation of state of matter at high density. The magnetic model Fe atmospheres produced in this paper are of sufficient quality to allow selection between $\mathrm{H}$ models and Fe models for pulsar $\mathrm{X}$-ray surface emission. If $\mathrm{H}$ atmospheres are preferred, the sophisticated modeling possible for this simple species will allow detailed interpretation of soft X-ray spectra. If however, Fe models remain acceptable, further careful study of the behavior of highly magnetized iron under neutron star surface conditions will be strongly motivated.

We thank G. G. Pavlov for supplying the sample magnetic $\mathrm{H}$ model spectra and for giving a careful and detailed critique of this paper. This work was supported in part by the National Sciences and Engineering Research Council of Canada (MR); by NASA grants NAG 5-3101, and NAGW-4526 (RWR); and by NAG 5-2868 and, through the Compton fellowship program, by NASA grant NAG 5-2687 (MCM).

\section{REFERENCES}

Becker, W. 1995, PhD Thesis, MPE Report 260

Bulik, T., \& Pavlov, G. G. 1996, ApJ, in press

Cox, John P., \& Giuli, R. Thomas 1968, Principles of Stellar Structure, (Gordon and Breach:New York)

Fehr, R. \& Kraft, W. D. 1995, Contrib. Plasma Phys., 35, 463

Ginzburg, V. L. 1970, The Propagation of Electromagnetic Waves in Plasmas, (Pergamon:Oxford)

Hernquist, L. 1984, ApJ Suppl., 56, 325

Hernquist, L. 1985, MNRAS, 212, 313

Hummer, D. G., \& Mihalas, D. 1988, ApJ, 331, 794

Khersonskii, V. K. 1987, Astr. Zh., 64, 433

Kopidakis, N., Ventura, J., \& Herold, H. 1996, A\&A, 308, 747

Meszaros, P. 1992, High Energy Radiation from Magnetized Neutron Stars, (University of Chicago Press:Chicago)

Meyer, R.D., Pavlov, G. G., \& Meszaros, P. 1994, ApJ, 433, 265

Mihalas, D. 1978, Stellar Atmospheres, (Freeman: San Francisco) 
Miller, M. C., \& Neuhauser, D. 1991, MNRAS, 253, 107

Miller, M. C. 1992, MNRAS, 255, 129

Neuhauser, D., Langanke, K., \& Koonin, S.E. 1986, Pys. Rev. A, 33, 2084

Page, D. 1995, ApJ, 442, 273

Pavlov, G. G., \& Meszaros, P. 1993, ApJ, 416, 752

Pavlov, G. G., Shibanov, Yu. A., Zavlin, V. E., \& Meyer, R. D. 1995, in The Lives of the Neutron Stars, ed. M. A. Alpar, Ü. Kiziloğlu, \& J. van Paradijs (Kluwer:Dordrecht) 71

Potekhin, A.Y., Pavlov, G. G., \& Ventura, J. 1997, A\&A, in press

Rajagopal, M. \& Romani, R.W. 1996, ApJ, 461, 327. (RR96)

Rogers, F. J. 1981, Phys Rev. A, 24, 1531

Rogers, F. J. 1986, ApJ, 310, 723

Romani, R. W. 1987, ApJ, 313, 718

Ruder, H., Wunner, G., Herold, H. \& Geyer, F. 1994, Atoms in Strong Magnetic Fields, (Springer-Verlag:Berlin)

Shibanov, Yu. A., Zavlin, V. E., Pavlov, G. G., \& Ventura, J. 1992, A\&A, 266, 313

Shibanov, Yu. A., Zavlin, V. E., Pavlov, G. G. \& Ventura, J. 1995, in The Lives of the Neutron Stars, ed. M. A. Alpar, Ü. Kiziloğlu, \& J. van Paradijs (Kluwer:Dordrecht) 91

Shibanov, Yu. A., \& Yakovlev 1996, A\&A, 309, 171

Zavlin, V.E., Pavlov, G.G. \& Shibanov, Yu. A. 1996, A\&A, 315, 141 (ZPS96) 